\title{
A Dry-Eye Warning System Based on the Estimation of the Human State Using a Single-Channel EEG Sensor
}

\author{
Kaoru Yoshii ${ }^{\mathrm{a}}$, Keiji Matsumoto ${ }^{\mathrm{a}^{*}}$

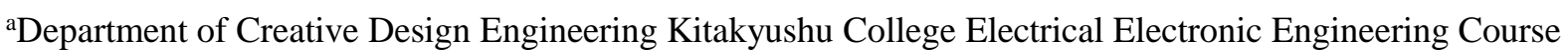 \\ 5-20-1 Shii, Kokuraminami, Fukuoka, 802-0985, Japan \\ "Corresponding Author:kmatsu@kct.ac.jp
}

\begin{abstract}
In this study, we designed and fabricated a warning system for suppressing dry-eye based on the estimation of the subject's state by using a single-channel EEG sensor. The system warns the subject if it decides that the dry-eye state occurs, based on the blinking rate reduction and the subject's concentration on some task. Brainwave data are used for estimating the subject's state, because these data contain a significant amount of relevant information. The Index of Brain Resting State (IBRS), calculated from the subject's EEG frequency spectrum, is used for estimating the subject's state. In addition, Dry-eye Diagnostic Chart (DDC), a novel classification method based on the IBRS and blinking rate, is proposed for estimating the dry-eye condition. The developed system provides a set of warning signals based on the RGB scheme.
\end{abstract}

Keywords: EEG, blink detection, IBRS, dry-eye.

\section{Introduction}

As a result of information and communications technology (ICT) development in recent years, efficient operations using a computer are routinely performed. Because the load of people engaged in these activities has been increasing year by year, dry eye, where the number of blinks dramatically decreases because of concentration, has become a problem. Dry eye, which is mostly regarded as having environmental causes, is believed to be a condition that impairs eye health as a result of a defect in tear production. There are many contributing factors for dry eyes, such as staring at a computer monitor and/or mobile phone and decreased indoor humidity as a result of air conditioning, which is commonly used in modern society. The secretion of tears is governed by the parasympathetic nerve, and decreases during a period of sympathetic dominance. Therefore, it is conceivable for the secretion of tears to be suppressed by various stresses. People have a tendency to think lightly of dry eye, because it is not a serious illness such as blindness. However, it might lead to chronic headache, systemic fatigue, and depression. Generally, dry eye is evaluated by a specialist using an optical instrument called a Tearscope Plus. Although research for the automatic diagnosis of dry-eye has been reported, all of them are strict medical diagnosis methods ${ }^{(1,}$ 2). Therefore, to the best of the authors' knowledge, there has been no report related to designing a simple warning system for healthy people to prevent dry eye.

In this study, the warning system for suppressing dry-eye based on the estimation of the human state by using a single-channel EEG sensor is designed and fabricated. When the symptom of decreased blinking is observed because a person is concentrating on their work, the system provides a warning to the subject. This system allows subjects to prevent dry eye by consciously increasing their blinking or adjusting the humidity of the room. To estimate the dry-eye condition, the system requires knowing the subject's state as well as the blinking rate. Therefore, the system does not use a camera; rather, the subject's state, including the dry-eye condition, is estimated by using only a single-channel EEG sensor.

\section{Material}

\subsection{EEG Sensor}

A large quantity of research on the gaze tracking of eye blinks using a camera has been reported ${ }^{(3-5)}$. Although 
it is possible to detect eye blinking without contact in this way, estimating the human state is very difficult. In this study, the human state and eye blinking are detected to predict the dry-eye state using an EEG sensor. The single-channel EEG sensor we used has two electrodes to measure brain potential: an active electrode and a reference electrode. The test subject wears the EEG sensor on their head, and it monitors the electrical potential between the electrodes attached to the forehead and earlobe. An EEG signal is presented in each frequency band-theta $(4-7 \mathrm{~Hz})$, low alpha $(7.5-9.25 \mathrm{~Hz})$, high alpha $(10-11.75 \mathrm{~Hz})$, low beta $(13-16.75 \mathrm{~Hz})$, and high beta $(18-29.75 \mathrm{~Hz})-$ once every second ${ }^{(6)}$. Spectral analysis of brain rhythms allows to determine which elements are dominant. All of the EEG signals, including both the time domain and frequency domain signals, were transmitted to an Arduino microcomputer via a Bluetooth wireless communication device. The time domain signal was used for determining the blinking rate, while the frequency domain signal was used for estimating the subject's state. The system provided warning signals based on the RGB scheme.

\subsection{Eye-blink detection}

A brain computer interface $(\mathrm{BCI})$ is a new technology that realizes communication between a human and machine without intentional action. An EEG signal is easily affected by artifacts because it is a weak signal on the order of a few microvolts. Although numerous methods to remove artifacts have been reported ${ }^{(7)}$, we attempted to use the blink artifact and EEG signal to detect dry eye. Figure 1 shows the raw brainwave in the time domain. The high amplitude shown in Fig.1 (2) represents the blink artifact. Generally, this is difficult to distinguish using only a threshold level because the amplitudes of artifacts from other actions in the region are sometimes higher than that of the blink. As an alternative to the threshold method, the method of correlation coefficients, which is used for comparing brainwave signals with a blink template, was employed in this study. It becomes possible to detect a blink by using this method, because the correlation coefficient between the template and a brainwave signal approaches a value of 1.0 if the two are similar.

$$
r_{c f}=\frac{\sum_{\text {data }=1}^{100}\left\{\left(x_{\text {data }}-\bar{x}\right)\left(y_{\text {data }}-\bar{y}\right)\right\}}{\sqrt{\sum_{\text {data }=1}^{100}\left(x_{\text {data }}-\bar{x}\right)^{2}} \sqrt{\sum_{\text {data }=1}^{100}\left(y_{\text {data }}-\bar{y}\right)^{2}}}
$$

The template and brainwave signals were compared by using the equation (1) : where $r_{c f}$ is the correlation coefficient, $\mathrm{x}_{\text {data }}$ is the recorded signal, $\mathrm{x}$ is average value of the recorded signal, $\mathrm{y}_{\text {data }}$ is the blink template, and $\mathrm{y}$ is average value of the blink template.

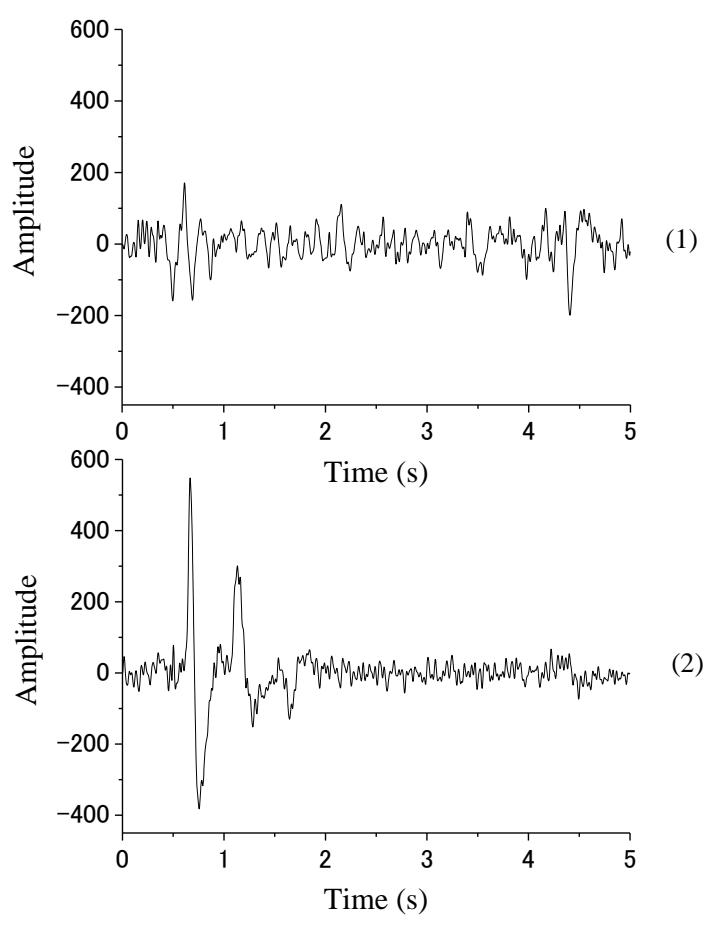

Fig.1 EEG signal in time domain (1) non blinking (2) blinking.

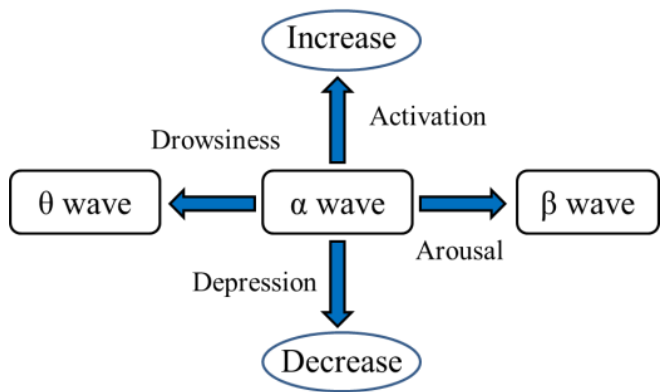

Fig. 2. Relationship between the human state and brainwave frequencies.

\subsection{IBRS}

IBRS, which is an electroencephalographic index to evaluate the resting state in a human, was proposed by Nakamura et al. ${ }^{(8)}$. Figure. 2 shows the conceptual diagram of the human state and brainwave frequencies. Generally, the alpha wave is used as an index of resting, and its integral amplitude value is ordinarily used as the 
discrimination criterion. However, other brainwave information is also needed to correctly estimate the human state, because alpha waves are considerably affected by visual information. Generally, the beta-wave element increases, along with its frequency, if the brain enters an excited state. In contrast, when the brain becomes drowsy and enters a suppressed state, the theta-wave element increases, and its frequency decreases. The condition between arousal and drowsiness is considered to be the brain-resting state. Therefore, the amplitude of the alpha wave compared to those of the theta and beta waves is defined as the IBRS. Formulas for the IBRS are defined as follows.

$$
\begin{gathered}
X_{I B R S}=\frac{\theta_{s t r}-\alpha_{s t r}}{\theta_{s t r}+\alpha_{s t r}} \\
Y_{I B R S}=\frac{\beta_{s t r}-\alpha_{s t r}}{\beta_{s t r}+\alpha_{s t r}}
\end{gathered}
$$

The IBRS is visually indicated in two dimensions using the ratio between the theta and alpha waves or between the beta and alpha waves (Fig.3). The horizontal axis is defined as the IBRS' $\mathrm{X}$ component, which is represented by both the theta and alpha waves, and the vertical axis is defined as the IBRS' Y component, which is represented by the beta and alpha waves. Both components of the IBRS are represented in a range of -1 to +1 . The value gradually becomes closer to -1 as the alpha wave increases. When the point $(-1,-1)$ is assumed to be the origin, the resting rate increases when the point indicated as the IBRS coordinate moves closer to the origin. The vector length from the origin is defined as the IBRS, as shown in Fig.3. We estimated the human state using this index.

\subsection{Dry-eye diagnosis chart}

We propose a dry-eye diagnosis chart (DDC) to estimate a human's dry-eye trend. This chart uses the number of blinks as the horizontal axis and the IBRS as the vertical axis, and the human state is divided into four sections (Fig.4). The first quadrant indicates that the human is in a relaxed mood with normal blinking. The state of a human in an active mood with normal blinking is shown in the second quadrant. There is no problem in both the first and second quadrants, because the human's blinking is normal. In contrast, both the third and fourth quadrants show decreased blinking, and the reason must be considered. In the fourth quadrant, it is assumed that even though the blinking is decreasing, the human is in a relaxed mood. In this case, the system does not have to give an alarm because the human might be drowsy. In the third quadrant, it is supposed that the decreased blinking is accompanied by an active mood. It is considered that this state could lead to a dry-eye condition. In this study, the third quadrant was the target area.

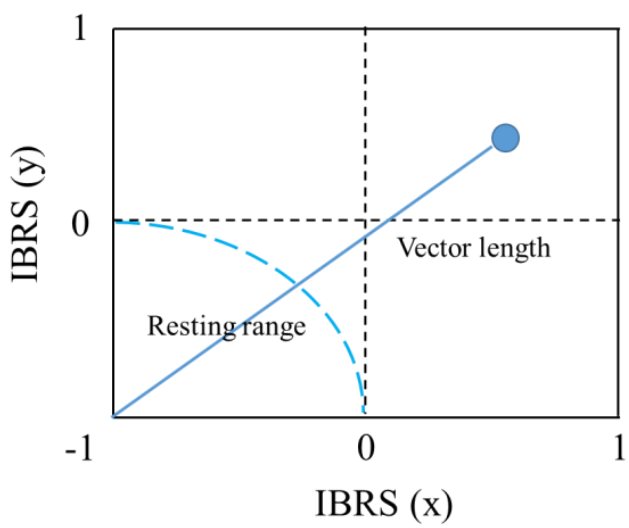

Fig. 3 2D display of the IBRS.

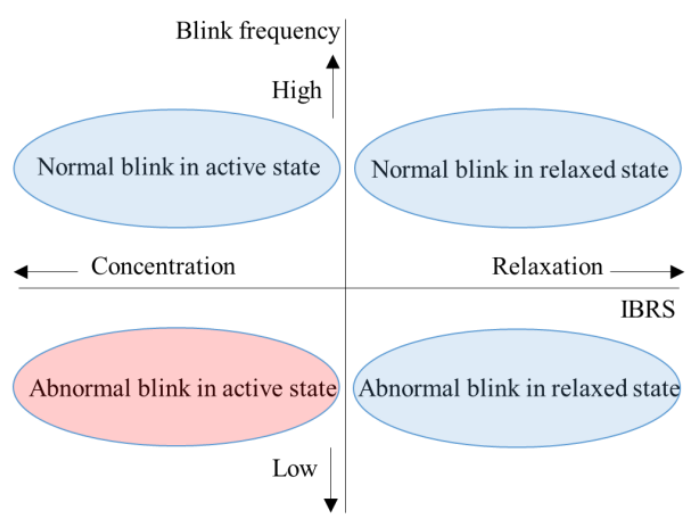

Fig. 4 Dry-eye diagnosis chart.

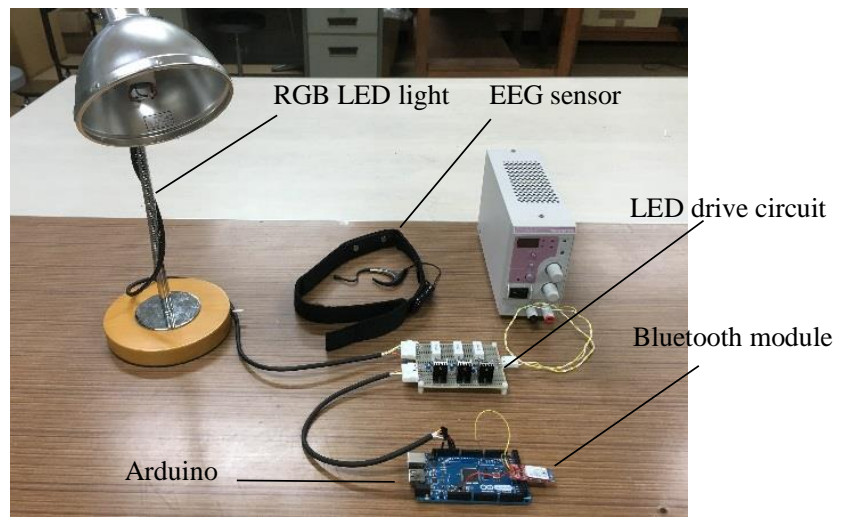

Fig. 5 Appearance of fabricated dry-eye system. 


\subsection{Fabricated dry-eye warning system}

Figure. 5 shows the designed and fabricated dry-eye warning system. The system consists of an EEG sensor, an Arduino microcomputer, a Bluetooth module, a LED and an LED drive circuit. The LED (OptoSupply, 6W, OSTCXBCBC1E), which contains an IC chip directly mounted on a substrate, was employed as the lighting equipment. The microcomputer estimated the dry-eye state given both the brainwave signal and the blink template, and simultaneously controlled the displayed LED colors via the LED drive circuit. When the microcomputer estimated that the subject was not in a dry-eye state, the LED light color was close to natural light. In the case of a suspected dry-eye state, the LED color changed to red, providing a clearly visible warning of the dry-eye state.

\subsection{Estimation method}

The estimation was carried out as follows. First, the warning system constantly observed the human's blinking. The IBRS was examined to estimate the human state as the next step, when the average number of blinks decreased. In a case where the IBRS value showed a relatively resting state, the state of the human was categorized as belonging in the fourth quadrant of the DDC. If the IBRS did not indicate a resting state, the state of the human was assumed to be in the third quadrant. The average value was employed because the IBRS normally fluctuated, along with the brainwaves.

\section{Result and discussion}

\subsection{Experimental result of the IBRS}

The dotted line in Fig. 3 shows the boundary between the third and fourth quadrants in the DDC. The X element of the IBRS has a negative value when the amplitude of the alpha wave is larger than that of the theta wave, and the $X$ element is close to zero if both of the amplitudes are approximately comparable. A positive value for the $\mathrm{X}$ element means the amplitude of the alpha wave is smaller than that of the theta wave. In the same way, the Y element of the IBRS has a negative value when the amplitude of the alpha wave is larger than that of the beta wave. The Y element has a positive value when the amplitude of the alpha wave is smaller than that of the beta wave. For these reasons, we assumed that positions on a curve with a radius of one constituted the boundary between the third and fourth quadrants in the DDC. To analyze the effectiveness of this diagnostic method, we performed the following experiments. First, an experiment was performed under the condition that the subject blinked less and progressively relaxed. Figure 6 shows the results of this experiment. Each point in Fig.6 is an IBRS value, which was calculated once per second, and $\mathrm{L}_{\mathrm{REF}}$ is the length computed using formula (4).

$$
L_{R E F}=\sqrt{\left(1+\frac{1}{n} \sum_{i=1}^{n} X_{i}^{I B R S}\right)^{2}+\left(1+\frac{1}{n} \sum_{i=1}^{n} Y_{i}^{I B R S}\right)^{2}}
$$

The $L_{\text {REF }}$ values obtained from the results of four subjects were $0.56,0.90,0.43$, and 0.67 . In each case, the value was inside of the boundary line. The next experiment was performed under the condition that the subjects focused on reading a book with less blinking. Figure 7 shows the results of this experiment. The $\mathrm{L}_{\mathrm{REF}}$ values obtained from the results of four subjects were 1.42, 1.43, 1.64, and 1.61. All the data were located outside of the boundary line. Based on these experimental results, it was found that there is a high possibility of the human state being in the third quadrant of the DDC if the $\mathrm{L}_{\mathrm{REF}}$ value is greater than one.

\subsection{Experimental result of the dry-eye warning system}

Evaluation of the developed dry-eye warning system was performed on a human subject wearing the brainwave sensor and switching between three different states every 5 min. As a first step, the fabricated system calculated both the number of blinks and the IBRS, and, given these data, calculated the dry-eye index. The dry-eye index defined in this study was calculated by using the fuzzy control method, based on both the number of blinks and the IBRS ${ }^{(9)}$. The dry-eye index was a number in the $0.0-1.0$ range. A dry-eye index close to 1.0 corresponds to a high probability of the subject being in the dry-eye state. The index value of $\sim 0.5$ signals that the subject is probably in a normal state with regard to dry eye. These treatments were conducted in real time, and the subject's state was estimated. Figures.8 (a) and (b) respectively show the number of blinks and the average IBRS, while Fig.8 (c) shows the dry-eye index. During the $5 \mathrm{~min}$ of the first part of the experiment, the subject relaxed with eyes closed. The experimental result in Fig.8 shows that, although a number of blinks were observed during the period of the first $30 \mathrm{~s}$, the fabricated 

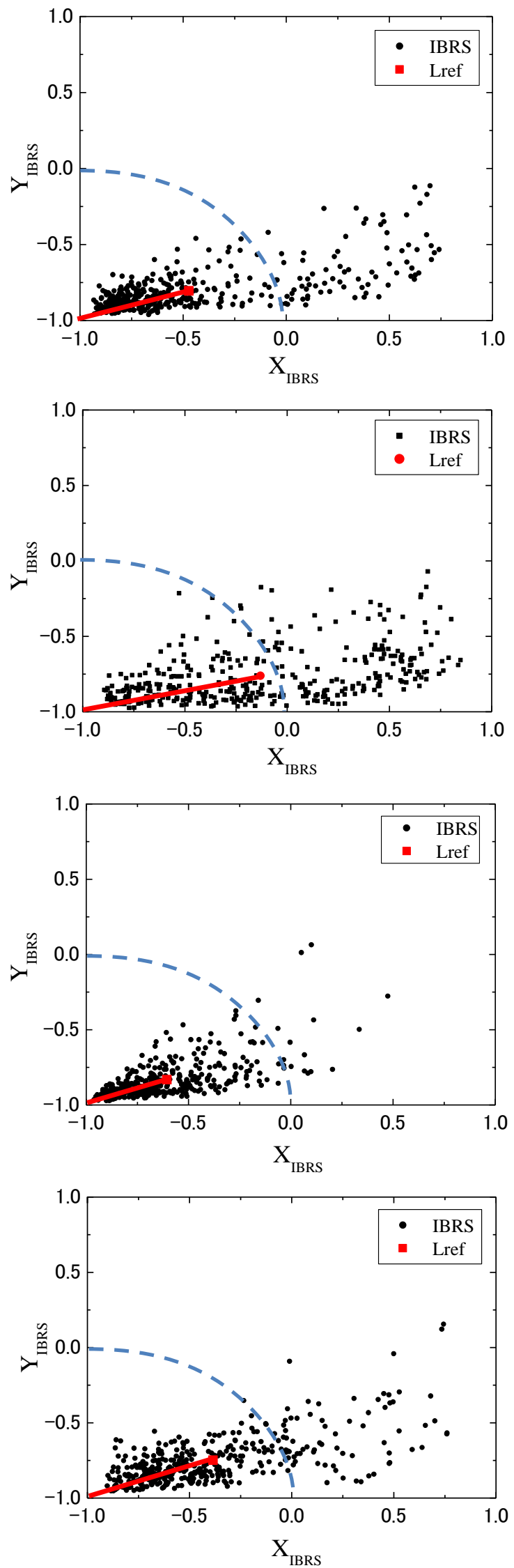

Fig. 6. The resting state of the subject with less blink.

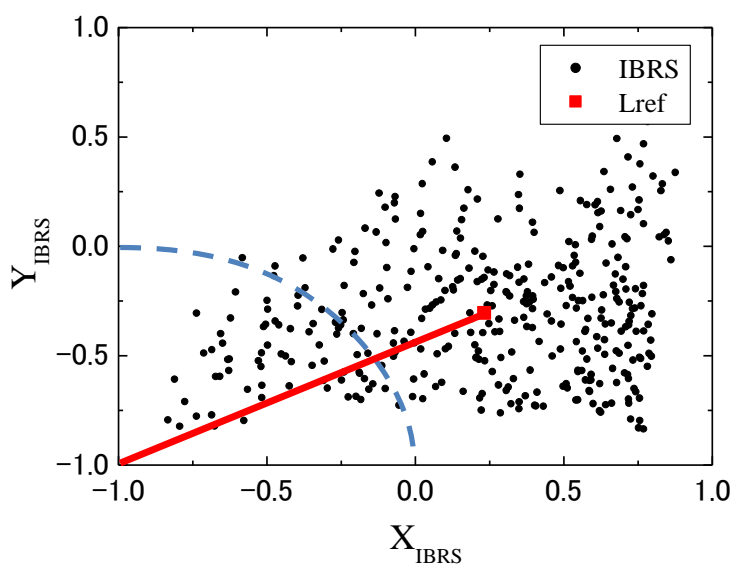

(1)

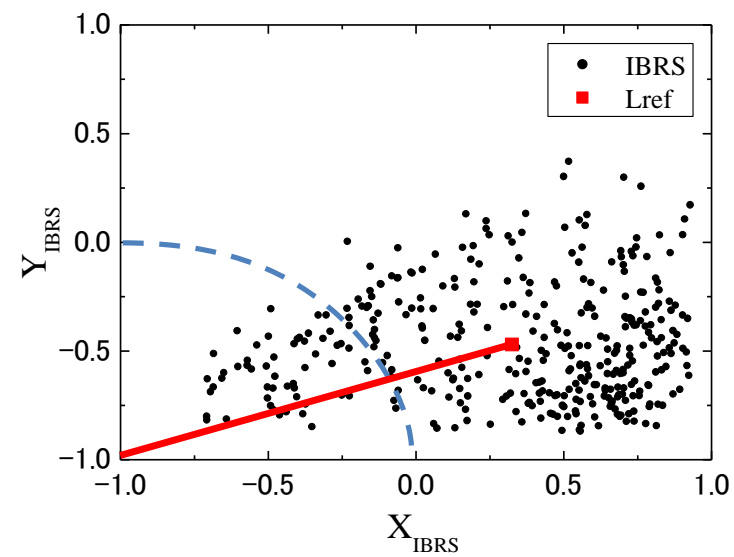

(2)

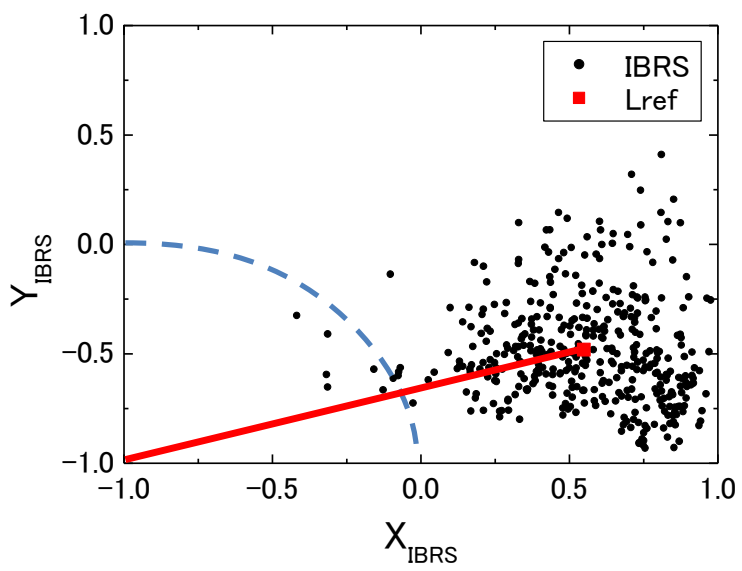

(3)

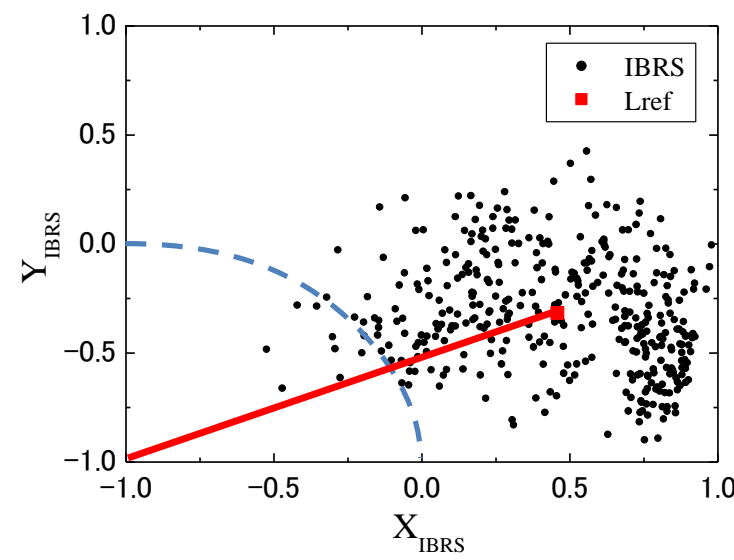

(4) 


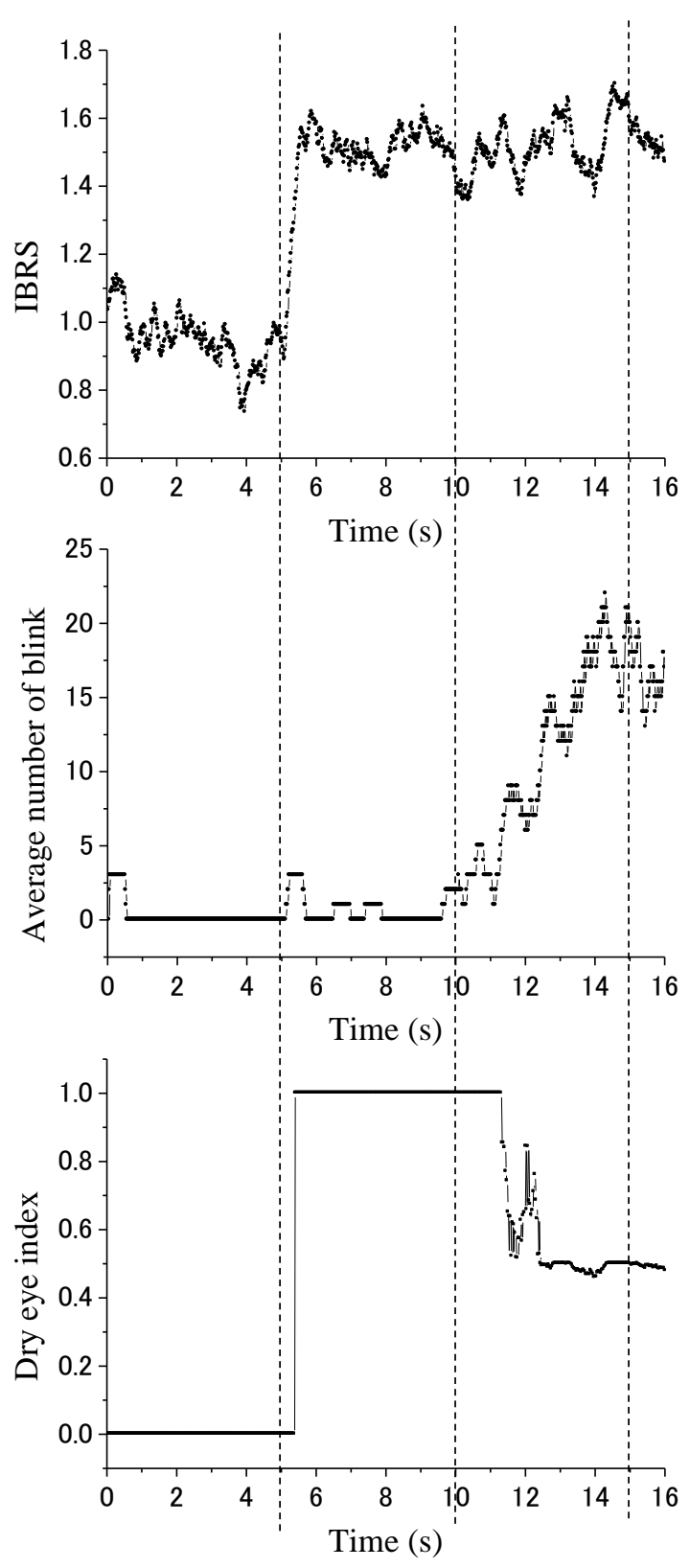

Fig. 8. Produced dry-eye index by treating IBRS and blink

(1) IBRS, (2) average number of blink, (3) dry-eye index.

system determined that the subject had almost no blinks. In the case of the IBRS, although these data exhibited a slight fluctuation, the system estimated that the subject was in the relaxed state, because the average IBRS was 0.95. This state, captured by the system, corresponds to the fourth quadrant in Fig.4. The dry-eye index calculated from these two results was $\sim 0.0$, implying a low probability of dry eye. During the next $5 \mathrm{~min}$ of the second section, the subject tried to refrain from blinking while reading a book. From the experimental result in Fig.8, it can be seen that the average value captured by the fabricated system during this period was less than once in every 30 seconds. In the case of the IBRS, the system estimated that the subject was active, based on the average value of 1.5. This state, captured by the system, corresponds to the third quadrant in Fig.4. The dry-eye index calculated from these two results was $\sim 1.0$, implying a high probability of dry-eye. During the 5 min of the last part, the subject had blinked normally while reading the book. From the experimental result in Fig.8, it can be seen that the average value of the number of blinks was approximately one every 2.8 seconds. In the case of the IBRS, the system estimated that the subject was active, because the average IBRS during this period was 1.51. This corresponds to the second quadrant in Fig.4. The dry-eye index that was calculated from these two results was $\sim 0.66$, implying a low probability of dry eye. From these results, we confirmed a good performance of the fabricated system that warns human subjects when conditions leading to the dry-eye state are sensed.

\section{Conclusions}

In this study, we designed and fabricated a warning system for suppressing the dry-eye condition based on the estimation of the human state by using a single-channel EEG sensor. The IBRS, calculated from the EEG frequency spectrum, was applied for estimating the human subject's state. In addition, the DDC, a novel classification method based on the IBRS and blinking rate, was proposed for estimating the dry-eye condition. The average IBRS values were $0.56,0.90,0.43$, and 0.67 for the active state and 1.42, $1.43,1.64$, and 1.61 for the relaxed state. The proposed system was able to distinguish the active state from the relaxed state. Moreover, the following results were obtained in the experiment for evaluating the fabricated system. When the subject was in the relaxed state with eyes closed, the dry-eye index was 0.0 , implying a low probability of being in the dry-eye state. When the subject read a book and refrained from blinking to the maximal possible extent, the dry-eye index was $\sim 1.0$, implying a high probability of dry eye. When the subject read a book while blinking normally, the dry-eye index was 0.66 . From these results, we confirmed a good performance of the fabricated system that provides a warning when sensing conditions leading to the dry-eye state. With the likely future development of simpler brainwave electrodes, the proposed system is expected to be an easy-to-use device for helping to prevent severe dry-eye syndrome. 


\section{References}

(1) B.Remeseiro, V.B.Canedo, D.P.Barral, A.A.Betanzos, B.G.Berdinas, A.Mosquera, M.G.Penedo, N.S.Marono : "A Methodology for Improving Tear Film Lipid Layer Classification", IEEE Journal of biomedical and health informatics, Vol.1, No.1, pp.1-9, Jan, 2013.

(2) T.Yedidya and R.Hartley: "Automatic Detection of Pre-Ocular Tear Film Break-Up Sequence in Dry Eyes", Digital Image Computing Techniques and Applications (DICTA), pp.442-448, Dec, 2008.

(3) F.Lu, Y.Sugano, T.Okabe, and Y.Sato : "Adaptive Linear Regression for Appearance-Based Gaze Estimation", IEEE Transactions on pattern analysis and machine intelligence, Vol.36, No.10, pp.2033-2046, Oct, 2014

(4) I.Bacivarov, M.Ionita, and P.Corcoran, "Statistical Models of Appearance for Eye Tracking and Eye-Blink Detection and Measurement", IEEE Transactions on Consumer Electronics, Vol. 54, No. 3, pp.1312-1320, Aug, 2008.
(5) J.W.Bang, E.C.Lee, and K.R.Park, New Computer Interface Combining Gaze Tracking and Brainwave Measurements", IEEE Transactions on Consumer Electronics, Vol. 57, No. 4, pp.1646-1651, Nov, 2011.

(6) N.Abe and K.Matsumoto : "Lighting control system using brainwave signals", Proc. ISTS 2013, pp.24-25, Nov, 2013.

(7) T.Okuyama, D.Kushida, A.Kitamura, A.Yao and H.Kondo : "Methodology of EOG Artifact elimination for EEG Signal by Using Correlation Coefficient and Independent Component Analysis", the 52th Japan Joint Automatic Control Conference, Nov, 2009.

(8) On the subject of brainwave : digital medic Inc, http://www.digital-medic.com/waves

(9) M.Hagiwara : "Neuro fuzzy genetic algorithm", Sangyo books, 1997, in Japanese. 
